Trauma Berufskrankh 2015 • 17:63 DOI 10.1007/s10039-015-0030-8

Online publiziert: 30. Mai 2015

๑) Springer-Verlag Berlin Heidelberg 2015

\author{
Dieter Rixen ${ }^{1,2} \cdot$ Barbara Herbst ${ }^{1,3}$ \\ ${ }^{1}$ Klinik für Orthopädie und Unfallchirurgie, Berufsgenossenschaftliche Unfallklinik Duisburg, \\ Duisburg, Deutschland \\ ${ }^{2}$ Fakultät für Gesundheit, Universität Witten/Herdecke, Witten, Deutschland \\ ${ }^{3}$ Sektion Rehabilitation, konservative Orthopädie \& Sportmedizin, Berufsgenossenschaftliches Zentrum \\ für Rehabilitation (BGZR), Berufsgenossenschaftliche Unfallklinik Duisburg, Duisburg, Deutschland
}

\title{
Berufsgenossenschaftliche Rehabilitation
}

Warum ist es uns wichtig, das Schwerpunktthema „berufsgenossenschaftliche Rehabilitation" in der Zeitschrift Trauma und Berufskrankheit ausführlich vorzustellen?

Zum einen hat die Rehabilitation im berufsgenossenschaftlichen Heilverfahren eine ganz besondere Bedeutung. Denn sie trägt mit ,allen geeigneten Mitteln" entscheidend dazu bei, Verletzten nach Arbeits- und Wegeunfällen möglichst schnell und effektiv den Weg zurück in den (beruflichen und privaten) Alltag zu ermöglichen. Zum anderen findet seit vielen Jahren eine enorme Weiterentwicklung in der berufsgenossenschaftlichen Rehabilitation statt - hin zu neuen, noch ausgereifteren Methoden und noch besseren Therapieformen. Ein wichtiges Ziel dieses Heftes ist es, einen Überblick über die zahlreichen berufsgenossenschaftlichen Heilverfahren und die dazu gehörigen Namensgebungen wie EAP, BOR, TOR, ABMR, Reha-AK, BGSW oder KSR zu geben sowie diese zu sortieren, zu definieren und einzuordnen.

Wann setzen wir im Klinikalltag diese verschiedenen Rehabilitationsmaßnahmen ein und welche fachliche Kompetenz decken wir ab?

Die in diesem Heft beschriebenen, regelhaft 4-wöchigen Reha-Maßnahmen sollen sicherstellen, dass die Wiedereingliederung der Patienten ins Erwerbsleben gelingt. Intensiv betreut werden neben den Unfallverletzten aus der Orthopädie und Unfallchirurgie auch handchirurgisch Verletzte und berufsgenossenschaftlich versicherte Hochleistungs- sportler. Für den Hochleistungssport werden von einem Mannschaftsarzt aus dem Profifußball Überlegungen zur Weiterentwicklung der Verfahren formuliert. Weiterhin wird in dieser Ausgabe dargestellt, dass der Schwerpunkt der berufsgenossenschaftlichen Rehabilitation zunehmend darauf abzielt, berufsbezogene Bewegungsabläufe sicher und dauerhaft wiederzuerlernen, idealerweise bei jedem Patienten mit individuell für den jeweiligen Beruf passenden Maßnahmen, ob Pflasterer, Dachdecker oder LKW-Fahrer.

Ein besonderes Instrument der berufsgenossenschaftlichen Rehabilitation sind sog. Reha-Fallkonferenzen. Diese werden durchgeführt, wenn die Rückkehr eines Unfallverletzten in seinen alten Beruf trotz aller Maßnahmen nicht möglich ist. In den Fallkonferenzen kommen Fachärzte mit den Reha-Managern der Unfallversicherungsträger und dem Patienten zusammen, um den aktuellen Stand des medizinischen Heilverfahrens festzustellen, ggf. noch erforderliche Behandlungsmaßnahmen zu veranlassen und die berufliche Wiedereingliederung/ Teilhabe auf den Weg zu bringen. Wichtige Langzeitergebnisse aus den Reha-Fallkonferenzen der letzten 5 Jahre stellen wir Ihnen erstmals im vorliegenden Heft vor.

Ergänzt wird das Themenspektrum dieser Ausgabe durch den aktuellen Stand der orthopädischen Schuhversorgung in der gesetzlichen Unfallversicherung.

Nach der Lektüre dieser Zeitschrift sind Sie auf dem aktuellen Stand der „berufsgenossenschaftlichen Rehabilitation"; viel Spaß beim Lesen!

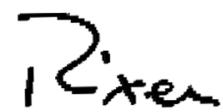

Prof. Dr. D. Rixen

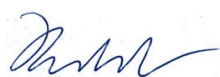

Dr. B. Herbst

\section{Korrespondenzadressen}

Prof. Dr. D. Rixen

Klinik für Orthopädie und Unfallchirurgie

Berufsgenossenschaftliche Unfallklinik

Duisburg, Großenbaumer Allee 250

47249 Duisburg

dieter.rixen@bgu-duisburg.de

\section{Dr. B. Herbst}

Sektion Rehabilitation, konservative Orthopädie \& Sportmedizin, Berufsgenossenschaftliches Zentrum für Rehabilitation (BGZR)

Berufsgenossenschaftliche Unfallklinik

Duisburg, Großenbaumer Allee 250

47249 Duisburg

barbara.herbst@bgu-duisburg.de 